\section{PSICOLOGIA IBEROAMERICANA}

\section{Psicología lberoamericana}

ISSN: 1405-0943

psicología.iberoamericana@uia.mx

Universidad Iberoamericana, Ciudad de

México

México

Sarudiansky, Mercedes

Ansiedad, angustia y neurosis. Antecedentes conceptuales e históricos

Psicología Iberoamericana, vol. 21, núm. 2, julio-diciembre, 2013, pp. 19-28

Universidad Iberoamericana, Ciudad de México

Distrito Federal, México

Disponible en: http://www.redalyc.org/articulo.oa?id=133930525003

- Cómo citar el artículo

- Número completo

- Más información del artículo

- Página de la revista en redalyc.org

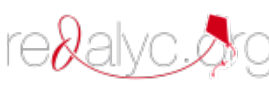

Sistema de Información Científica

Red de Revistas Científicas de América Latina, el Caribe, España y Portugal

Proyecto académico sin fines de lucro, desarrollado bajo la iniciativa de acceso abierto 


\title{
Ansiedad, angustia y neurosis. Antecedentes conceptuales e históricos
}

\author{
Anxiety, Angustia and Neurosis. A Conceptual and Historical Background
}

\author{
Mercedes Sarudiansky ${ }^{1}$ \\ Consejo Nacional de InVestigaciones Científicas y Técnicas \\ FACUltad de Psicología \\ UniversidAd de BUenos Aires
}

\section{RESUMEN}

La ansiedad es uno de los conceptos más estudiados e investigados en el campo de la salud mental, sobre el cual algunos autores han destacado su carácter multívoco y ambiguo. En este artículo se realiza un recorrido etimológico y conceptual sobre la ansiedad, intentando reflejar la variabilidad de contenidos que presenta este término en la actualidad, en sus distintos planos de abordaje. En primer lugar, se hace referencia a los antecedentes lingüísticos del vocablo "ansiedad", destacando las problemáticas surgidas a partir de la traducción de términos clave. En segundo lugar, se da cuenta de la perspectiva de distintos referentes de la psicopatología occidental sobre este concepto, así como de los criterios de clasificación propuestos. Posteriormente, se señala la relación del concepto de neurosis con las descripciones actuales sobre la ansiedad. Para finalizar, se destaca la importancia de considerar estas cuestiones tanto en el área clínica como en los terrenos académicos y de investigación.

Palabras clave: ansiedad, angustia, neurosis, descripciones, psicopatología.

\section{ABSTRACT}

Anxiety is one of the most frequently studied and researched topics in the field of mental health and some authors have focused on its multivocal and ambiguous nature. This article studies the etymological and conceptual aspects of anxiety, in order to reflect the variability of contents that are currently described by the various usages of this term. Firstly, we refer to the linguistic background of the term "anxiety", in particular the problems arising from the translation of key words. Secondly, we examine the perspective of various referents of western psychopathology in regard to this concept, as well as the proposed classification criteria, before identifying the relationship between the concept of neurosis and the current descriptions of anxiety. Finally, we highlight the importance of considering these questions both in terms of clinical treatment and in relation to the fields of academia and research.

Keywords: anxiety, distress, neurosis, descriptions, psychopathology.

\footnotetext{
${ }^{1}$ Recibido: 21 de noviembre, 2013 - Aceptado: 6 de agosto, 2013.

Correspondencia: Mercedes Sarudiansky, Guardia Vieja 4357, PB 5, Ciudad Autónoma de Buenos Aires, Argentina, C. P. 1192.
} 


\section{INTRODUCCIÓN}

La ansiedad es, sin lugar a dudas, uno de los conceptos centrales en el ámbito de la salud mental. Sin embargo, ello no implica que se trate de una categoría simple y uniformemente definida, sino que ya desde sus bases etimológicas y de sus antecedentes conceptuales, teóricos y disciplinares podemos dar cuenta de que es un constructo difuso, ambiguo y complejo (Jablensky, 1985; Lewis, 1980). Muchas veces estas características no son consideradas por investigadores, académicos y profesionales de la salud mental; por este motivo, la explicitación de estas cualidades es un aspecto importante a tener en cuenta para que los fenómenos como la ansiedad puedan ser entendidos y estudiados en toda su extensión.

El objetivo del presente trabajo es presentar un recorrido etimológico y conceptual sobre la ansiedad, que sustenta la variabilidad de contenidos de este término, en la actualidad, tanto en el plano técnico-profesional como del lenguaje cotidiano. Se iniciará haciendo referencia a los antecedentes lingüísticos del vocablo, para luego dar cuenta de las cualidades o las características que se incluyen en este concepto. Posteriormente, se expondrá cómo lo abordaron diferentes autores - referentes de la psicopatología occidental—, ya sea desde su fenomenología - en particular, a partir de su relación con el miedo- como desde la propuesta de cuadros diagnósticos discretos. A continuación, se plantea un foco en el concepto de neurosis, el cual demuestra ser uno de los eslabones clave para la definición de muchos de los criterios diagnósticos utilizados en la psicología actual, entre ellos los que se refieren, contemporáneamente, como "trastornos de ansiedad". Este recorrido permitirá dar cuenta de la heterogeneidad sintomática y fenomenológica que caracteriza a la ansiedad en los criterios diagnósticos actuales, cuestión que se evidencia de manera concreta a partir de los diferentes cuadros y enfermedades que presentan distintos autores como antecedentes directos de esta categoría, y que se refleja en la diversidad de teorías explicativas coexistentes en los ámbitos de salud mental contemporáneos.

\section{LOS VOCABLOS "ANSIEDAD" Y "ANGUSTIA"}

Desde un punto de vista etimológico, la palabra "ansiedad" tiene un antepasado directo en latín, anxietas. Este término latino proviene, a su vez, de la raíz in- doeuropea angh de la cual se deriva no sólo - y aquí está lo interesante- el vocablo anxietas, sino también la palabra ango, angustia. Ambos términos, como lo señalara Pichot (1999), eran utilizados de manera indistinta para hacer referencia a diferentes significados, tal como puede rastrearse en textos de autores clásicos como Cicerón o Virgilio: constricción —angostamiento, achicamiento- y sufrimiento. Y es justamente esta ambigüedad de significados y de términos (ansiedad/ angustia; constricción/sufrimiento) la que atravesará toda la historia del concepto y que llegará hasta nuestros días.

La distinción ansiedad/angustia, especialmente cuando nos referimos a terminología técnica psicológico-psiquiátrica, guarda especial interés para la lengua castellana, puesto que ambos términos hacen referencia a trastornos, cuadros o sintomatología propias de estas disciplinas psi. El francés también posee la misma dualidad: anxiété/angoisse. Por el contrario, la utilización de vocablo equivalente a angustia en el idioma inglés no posee el mismo peso. El término anxiety es el depositario de todas las elucidaciones conceptuales psi, así como el término angst lo es para el alemán. Este hecho dará pie a discusiones en torno a la distinción o indistinción de estos conceptos (en español y francés), dadas las confusiones derivadas de los trabajos de traducción. Encontramos, pues, una asimetría terminológica en relación con la ansiedad y la angustia en español, respecto del alemán y del inglés —dos de las principales fuentes de teorías y praxis en psiquiatría, psicología y psicoanálisis-, no así en francés. De esta manera, es esperable que encontremos diferencias en relación con el origen de las fuentes: mientras que en los textos traducidos del francés es común hallar aclaraciones respecto de la equivalencia o no de ambos términos, esta disquisición no se encuentra con la misma facilidad en los textos anglosajones (a excepción de, por ejemplo, Lewis, 1980 o Barlow, 2002).

Un claro ejemplo de ello es el célebre diccionario de medicina editado por Littré y Robin (en Berrios, 2008), quienes definieron angoisse como "sensación de constricción o de presión en la región epigástrica, acompañada de una gran dificultad para respirar y de inmensa tristeza; éste es el grado más avanzado de la ansiedad". Mientras que anxiété se correspondía con "un estado afligido y agitado, con sensación de difi- 
cultad respiratoria y presión sobre la región precordial; inquietud, ansiedad y angustia eran tres etapas del mismo fenómeno". Desde esta perspectiva, la diferenciación entre tres términos - ansiedad, angustia e inquietud - tiene un carácter dimensional, esto es, se distinguen en virtud de su grado de severidad o de capacidad de generar sufrimiento al individuo.

Por su lado, Hardy, Hardy y Kerneis (1985) remiten a la distinción propuesta por Brissaud en 1890, quien definía a la angustia como un trastorno físico caracterizado por una sensación de constricción, mientras que la ansiedad se correspondía con un trastorno psíquico que contiene sentimientos de inseguridad. De todas maneras, en el posterior desarrollo del artículo de Hardy et al. (1985) no existe una diferenciación conceptual entre ambas locuciones. Contrariamente a estas perspectivas, Ey (2008) destaca la postura de Boutonier, quien, siguiendo la tradición humanista, no distingue entre ansiedad y angustia. De todas maneras, Ey hace referencia al uso cotidiano de los términos, reservando el de angustia para la vertiente física de la emoción. Sin embargo, y de manera explícita, empleará ambos de manera indistinta durante toda su obra.

Dentro de las vertientes anglosajona y germánica, se encuentra una observación de Strachey (1999); en su introducción a uno de los primeros escritos freudianos acerca de la angustia - "Sobre la justificación de separar de la neurastenia un determinado síndrome en calidad de neurosis de angustia" - señala que Freud, si bien en sus obras en alemán distingue entre los vocablos angst (angustia), furcht (temor) y schreck (terror), en sus escritos en francés utilizó como sinónimos angoisse y anxiété para traducir el término angst. Esta cuestión corrobora, en parte, lo que planteamos al principio respecto de la diferencia entre la perspectiva franco-española, que posee dos términos técnicos, de la alemana y la inglesa, que tienen únicamente uno.

Como se ha podido observar, esta asimetría no sólo se desprende de la cantidad de palabras técnicas disponibles en uno u otro idioma, sino también de la cantidad o la calidad de acepciones que cada uno de los autores le atribuyó a los términos. De esta manera, a pesar de que, por ejemplo, del alemán al inglés sólo habría una opción de traducción del vocablo angst -anxiety -, ciertos autores, como Lewis (1980), dan cuenta de la inexactitud de tal correspondencia. Así, sugiere, como en el caso de la obra de Kierkegaard, que expresiones tales como pavor, agonía, terror o miedo serían acepciones más correctas para traducir al término angst al inglés. De este modo, la vertiente terminológica de la ansiedad presenta diferentes vocablos, los cuales pueden funcionar tanto como sinónimos - especialmente en el terreno de lo coloquial-, aunque muchas veces poseen caracteres específicos que los diferencian unos de otros. De todas maneras, esta especificidad, sea por las dificultades relativas a la traducción o por las complejidades propias de cada lengua, muchas veces se encuentra poco establecida, produciéndose, de este modo, indistinciones en el plano del lenguaje técnico o conceptual del ámbito psi.

\section{LAS MANIFESTACIONES DE LA ANSIEDAD EN PSICOPATOLOGÍA}

Berrios (2008) explica que las conductas "de tipo ansioso" - "inquietud y desasosiego", según el autorpueden ser rastreadas en la bibliografía de distintos momentos históricos. En efecto, desde los primeros textos atribuibles a la disciplina médica occidental, se hacía referencia a estados de angustia o aflicción que acompañaban el curso de distintas enfermedades físicas, aunque no necesariamente eran circunscriptos a cuadros mórbidos específicos. Por ejemplo, en el siglo v a. C., en Aforismos y sentencias Hipócrates señalaba sobre un enfermo: "En el [día] tercero estuvo muy caído, tuvo sed, congoja en el estómago, mucha inquietud y aflicción con angustias, deliró" (enfermo vigesimosegundo). Asimismo, tal como plantean Papakostas, Eftychiadis, Papakostas y Christodoulou (2003), también pueden observarse condiciones homologables a estados de ansiedad en textos de Sófocles, Platón o Arateo.

Ya entrado el siglo xIx, Pinel incluía en distintos cuadros los síntomas que actualmente se corresponderían con la ansiedad, tales como la epilepsia, la melancolía o ciertas neurosis, al igual que Georget (Beer, 1996). Más adelante Ey, en su introducción al estudio sobre la ansiedad mórbida, da cuenta de ella como una "reacción afectiva patológica tan general que, lejos de constituir un síntoma o un síndrome bien delimitado, se encuentra más o menos en la estructura misma de todos los estados psicopatológicos coloreándolos así de uno de los reflejos más específicos del alma humana, 
la inquietud y el miedo" (Ey, 2008). Así, se evidencian ciertas coincidencias con la postura de Pinel, pues, más que ser un cuadro específico, complementa enfermedades más complejas, mientras que, a su vez, nos da pie para relacionar de manera concreta a la ansiedad con el miedo y la inquietud.

Éste es un punto que reviste una suma importancia, ya que en la mayoría de los textos contemporáneos que tratan sobre la ansiedad, se hace referencia, las más de las veces, a su relación con el miedo. Al miedo como una experiencia propia de los seres humanos - aunque no exclusiva-, la cual implica ciertas consecuencias, ya sean éstas comportamentales, fisiológicas, emocionales, psicológicas, cognitivas o espirituales.

\section{MIEDO, ANSIEDAD Y LA DISTINCIÓN ENTRE NORMALIDAD Y PATOLOGÍA}

Cuando hablamos de miedo nos referimos a una emoción o una respuesta psicológica, comportamental o fisiológica, respecto de un estímulo potencialmente peligroso. Su existencia se asocia con un circuito de defensa filogenéticamente antiguo - esto es, desde un punto de vista darwiniano, compartido con organismos incluso más primitivos en la escala evolutiva- que posibilitó a las distintas especies hacer frente a predadores o amenazas y, por ende, a sobrevivir, puesto que pone en movimiento mecanismos de huida, paralización o lucha (Cannon, 1927). De manera descriptiva, estas respuestas del organismo frente a las amenazas se podrían ordenar en distintos niveles: en el fisiológico, la activación de ciertos mecanismos biológicos que involucran al sistema nervioso central, cardiovascular, neuroendocrino, inmunológico, digestivo, entre otros, los cuales serían responsables de respuestas tales como el aumento del ritmo cardiaco, la vasoconstricción en extremidades, la aceleración de la respiración, la sudoración y la dilatación de las pupilas. Estas respuestas son coherentes con los comportamientos que se asocian a la lucha o la fuga, como por ejemplo las necesidades básicas implicadas en correr o combatir (para lo cual es necesario un aumento del torrente sanguíneo, así como también mayor cantidad de oxígeno en sangre), evitar lastimaduras graves (la vasoconstricción de las extremidades impediría el sangrado excesivo en estas partes del cuerpo que más expuestas están) o la agudeza perceptiva e hipervigilancia. En el nivel psi- cológico, el miedo se encuentra relacionado con una sensación de aprensión, de inquietud, nerviosismo e incertidumbre. Existe cierto consenso en considerar que este tipo de reacciones son universales, esto es, están presentes en todos los seres humanos, e incluso en la mayoría de las especies vertebradas. Ello se evidencia, en la práctica, a partir de la existencia de investigaciones sobre respuestas al miedo basadas en modelos animales, en referencias de tales reacciones en la antigüedad e, incluso, en contextos distintos al mundo occidental -como, por ejemplo, en los documentos de la medicina tradicional china, donde el miedo está incluido como una de las cinco emociones fundamentales (Hsu, 1999).

De este modo, se tiene una emoción o reacción - el miedo - a la cual se le puede atribuir cierto carácter universal, que involucra una activación en distintos niveles del organismo a partir de la presencia de un peligro (ya sea éste una situación, un predador o un objeto). Teniendo en cuenta la relación que hay, en la actualidad, entre la ansiedad y el miedo - particularmente, en función de la pertinencia del miedo como uno de los elementos básicos para la definición de los trastornos de ansiedad-, se puede afirmar que estas cualidades atribuibles a él también deberían estar presentes en una definición de la ansiedad.

Sin embargo, la relación entre miedo y ansiedad tampoco ha sido demasiado clara. Freud, por ejemplo, diferenciaba la angustia - angst - del miedo - furcht- y del terror - schreck- (véase supra), señalando que lo que distingue a la angustia del miedo se basa en la anticipación del peligro, por lo que no es necesaria la presencia real del objeto amenazante, mientras que el terror se caracteriza por la intensidad de la reacción. Jaspers (1998) también hacía esta distinción, sosteniendo que el miedo se encuentra orientado hacia algo o alguien, mientras que la ansiedad no presenta un objeto determinado. Contrariamente, Pichot (1999) señala que en realidad la lengua alemana no hace tal distinción, ya que el vocablo angst significa tanto miedo como ansiedad, por lo cual, según él, es una paradoja que los autores alemanes sean los que más diferencien ambos términos.

Sin embargo, esta discriminación no ha tenido un consenso homogéneo en el ámbito psicológico-psiquiátrico. Desde tiempos tempranos, distintos autores, 
como por ejemplo Storring (Ey, 2008), ya concluían que el miedo es una forma especializada de la angustia/ansiedad. De hecho, dentro del abanico que engloba las reacciones que podrían ser catalogadas como "ansiosas", aquellas referentes al miedo desempeñan un papel destacado, pero debemos tener en cuenta que el constructo ansiedad/angustia es más complejo aún.

En un principio, basándose en el uso popular de tales palabras en español, se puede observar que el miedo, en general, hace referencia a una reacción negativa, no deseada. Sentir miedo, pese a sus características más o menos adaptativas, no es algo que las personas deseen tener. Con la ansiedad, por el contrario, se encuentra una ambigüedad en cuanto a su contenido connotativo. Este término puede ser utilizado de manera positiva: sentir ansiedad, en muchos casos, es visto como equivalente a tener expectativas, a la ilusión de algo que está por venir; por otro lado, también se encuentra asociada a tecnicismos psicológico-psiquiátricos. En ciertos contextos, "tener ansiedad" o "ser ansioso/a", lejos de poseer una connotación positiva como se mencionó previamente, se relaciona más con patología y enfermedad que, incluso, el miedo. De la misma manera, la angustia también se encuentra muy ligada a términos técnicos propios de las disciplinas psi.

Desde su connotación negativa, se observa a su vez una vertiente ligada con la patología y otra asociada a los fenómenos "normales". La distinción entre uno y otro es una cuestión que se sigue discutiendo hoy en día.

De esta manera, nos encontramos con un término - ansiedad- que etimológicamente se relaciona con otro - angustia-, pero que de manera descriptiva se asocia al miedo, al nerviosismo y a ciertos estados patológicos, ya sea como un cuadro nosológico más o menos completo, o en forma de signos o síntomas que complementan a otros trastornos. En ambas vertientes -la que llamamos "positiva" y aquella más ligada con la patología-, la ansiedad se relaciona con un aumento de la tensión del organismo, ocasionado por la activación - psíquica y/o biológica - frente a la presencia de un peligro (real, imaginado o anticipado) o de una expectativa de diversa índole, lo cual trae como consecuencias un gran abanico de sensaciones, emociones y cogniciones difíciles de delimitar o de categorizar. Ello hace a la inespecificidad del constructo ansiedad. Sin embargo, desde el campo de la psicopatología se han realizado intentos de delimitar qué síntomas se asocian con la ansiedad y cuáles no.

\section{CLASIFICACIONES EN PSICOPATOLOGÍA SOBRE LA ANSIEDAD}

Así pues, desde hace más de cien años se han hecho intentos para clasificar y tipificar los síntomas que se asocian con la ansiedad/angustia. En un artículo de 1894, Freud ya hacía referencia a las distintas características de las neurosis de angustia, entre las que incluye: irritabilidad general, expectativa angustiada, la posible presencia de "ataques de angustia", los cuales se caracterizan por perturbaciones de la actividad cardiaca (palpitaciones, arritmia, taquicardia), perturbaciones de la respiración (disnea nerviosa, ataques semejantes al asma, etc.), oleadas de sudor, temblores y estremecimientos, ataques de hambre, diarreas, terror nocturno, perturbaciones digestivas, vértigo, congestiones y parestesias (Freud, 1999).

Ey, al referirse a la ansiedad como un "modo de organización catastrófica, frente a un peligro a veces presente e inminente, pero a veces ausente e incluso imaginario" (Ey, 2008), señala que para su descripción deben tomarse en cuenta, fundamentalmente, tres aspectos: la amplificación peyorativa, que se refiere a la exageración de las perspectivas pesimistas ligadas con la desgracia; la espera del peligro futuro e inminente, que puede incluir el miedo a la muerte, al mal, al dolor, a la tortura, etc.; y, por último, el desasosiego, el sentido agónico de la existencia.

Según los sistemas de clasificación vigentes (American Psychiatric Association, 2000; Organización Mundial de la Salud, 1992), los trastornos de ansiedad se organizan en diferentes cuadros, que incluyen desde estados paroxísticos ansiosos - los ataques de pánico o crisis de angustia - hasta trastornos que se definen por la presencia de temores irracionales o preocupaciones excesivas, que no necesariamente incluyen la sintomatología aguda.

Otro de los puntos clave a la hora de definir la ansiedad tiene que ver con el hecho de si ésta es un fenómeno circunstancial (es decir, agudo, transitorio) o permanente (de carácter crónico). Por ejemplo, uno de los instrumentos psicométricos más utilizados para la evaluación de la ansiedad, el Inventario de Ansiedad 
Rasgo-Estado (Spielberger, Gorsuch \& Lushene, 1970), da cuenta de esta distinción, señalando que la ansiedadestado supone un estado emocional transitorio, mientras que la ansiedad-rasgo se explica a partir de lo que sería una "tendencia ansiosa", propia de un rasgo de personalidad.

En cuanto a la sintomatología que caracteriza a la ansiedad, Kristensen, Mortensen y Mors (2009) encontraron 110 síntomas corporales y 77 síntomas emocionales y cognitivos, cuestión que evidencia que el concepto de ansiedad presenta la capacidad de ser permeable a la inclusión o exclusión de todo tipo de sintomatología.

Ahora bien, esta particularidad tendrá distintas consecuencias: por un lado, una exuberante cantidad de investigaciones, ya sea en ciencia básica o aplicada, en torno al estudio de sus distintas manifestaciones; otra inagotable suma de investigaciones en torno a sus posibles dispositivos de atención; una gran diversidad de teorías sobre sus causas y mecanismos desde distintas perspectivas (biológicas, etiológicas, psicológicas, sociales, religiosas, etc.). A su vez, debido a esta inespecificidad es posible asociarla con otros conceptos tales como las neurosis - sobre lo cual nos detendremos a continuación-, la histeria, el estrés, las fobias, así como otras enfermedades no necesariamente reservadas al campo de las disciplinas "psi", como las cardiopatías, el cáncer, los trastornos digestivos, etcétera.

\section{ANSIEDAD Y NEUROSIS}

Distintos autores ubican a la neurosis como uno de los conceptos clave que nos ayudará a profundizar nuestro recorrido (Conti \& Stagnaro, 2007; Berrios, 2008). El término fue introducido, formalmente, en la literatura médica occidental por el médico escocés William Cullen en 1769, haciendo referencia a "enfermedades de los sentidos y del movimiento, sin fiebre idiopática y sin afección local" (Cullen, 1783). De esta manera, la distinguía de otras clases de enfermedades agrupadas bajo los nombres de fiebres o pirexias (entre las cuales se encontraban la neumonía, gastroenteritis, hepatitis, malaria, urticaria), caquexias (que incluía sífilis, escorbuto, tricoma), y locales (donde se agrupaban bulimia, polidipsia, ninfomanía, anorexia, nostalgia, afonía, estrabismo). Este autor se basó en la metodo- logía propuesta por Sydenham ${ }^{2}$ para realizar su taxonomía de las enfermedades. La justificación para la introducción de esta categoría se basó en la necesidad de superar la inexactitud del concepto de "enfermedad nerviosa", ${ }^{3}$ puesto que "casi todas las alteraciones mórbidas del cuerpo, hasta donde se puede entender, dependen de tal manera de las mociones del sistema nervioso, que casi todas las enfermedades podrían, en este sentido, ser denominadas nerviosas" (Cullen, 1808). A su vez, dentro del grupo de las neurosis encontramos cuatro categorías u órdenes principales: las "comatas", que suponían una disminución de los movimientos voluntarios, con sueño o una suspensión de los sentidos, las cuales incluían a la apoplejía y las parálisis; las "adynamiae", que implicaban una disminución de los movimientos voluntarios, cuyos cuadros principales eran el síncope, la dispepsia, la hipocondriasis y la clorosis; en tercer lugar los "spasmi" o espasmos, esto es, movimientos irregulares de los músculos, entre los que se encontraban el tétanos, las convulsiones, corea, epilepsia, palpitaciones, asma, disnea, cólera, diarrea, diabetes, histeria e hidrofobia; y finalmente, las "vesaniae", un trastorno de las funciones de las facultades mentales de juicio, sin fiebre ni somnolencia, donde se ubicaban la amentia, la melancolía, la manía y la onirodinia.

Como puede observarse, en la denominación de "neurosis" aparece una gran diversidad de cuadros, algunos de los cuales sería discutible que en la actualidad se rotulen de esta manera. De todos modos, tal distinción tuvo un gran impacto en la medicina de la época, e incluso en uno de los médicos que más influencia tuvo en el desarrollo de las disciplinas psicológico-psiquiátricas, como Philippe Pinel (1745-1826). Sin em-

\footnotetext{
${ }^{2}$ Thomas Sydenham (1624-1689) fue uno de los médicos más reconocidos de la Inglaterra del siglo xvir. Es considerado como uno de los precursores de los sistemas diagnósticos actuales, puesto que propuso que las clasificaciones mórbidas no se basen en teorías especulativas, sino más bien en descripciones clínicas precisas. Tomó como modelo las clasificaciones botánicas, sosteniendo que debe tenerse en cuenta el mismo nivel de detalle. Asimismo, fue uno de los precursores en lo que hace a relacionar diagnóstico y pronóstico de las enfermedades (Balint, Buchanan \& Dequeker, 2006).

${ }^{3}$ De todas maneras, la denominación "enfermedades nerviosas", a pesar de la aparición del concepto de neurosis, siguió utilizándose hasta la actualidad. El taxón "nervios” es una categoría que aún sigue vigente y que, de hecho, ha generado discusiones en torno a su relación con los trastornos de ansiedad según el DSM-IV TR (2000).
} 
bargo, esta acepción del término no se mantuvo intacta a lo largo de los siglos que suponen su existencia. Por ejemplo, Georget (1795-1828) las redefinió como enfermedades crónicas e intermitentes, sin lesión anatómica post mortem, no necesariamente peligrosas, pero que ocasionaban excesivo sufrimiento en quienes las padecían. Según este autor, las neurosis podían incluir dolores de cabeza frecuentes, locura, hipocondría, catalepsia, corea, histeria, palpitaciones nerviosas, asma, gastralgia y neuralgias (Beer, 1996). Beer señala que la predominancia del método anatomo-clínico, en la medicina del siglo xIX, propició la profundización de esta distinción entre enfermedades funcionales y aquellas con cierta base anatómica, lo cual ubicaba a las neurosis más en el campo de la neurología que en la psiquiatría. De todas maneras, la discusión en torno a la localización anatómica o no de ciertas enfermedades "neuróticas" es un tópico que aún sigue vigente.

La cualidad de "funcional" no resulta útil para delimitar un grupo más o menos circunscripto de enfermedades, ya que, como señala Køppe (2009), existen diferentes maneras de utilizar tal término. Por ejemplo, uno de los usos más comunes durante el siglo XIX suponía que para que una enfermedad sea considerada funcional no debía haber evidencia de explicación anatómica o fisiológica. Desde esta perspectiva, las neurosis serían entidades transitorias, las cuales perderán su cualidad en el momento en que se encuentre su explicación somática. Otra postura pone en primer plano la oposición anatomía-fisiología, análoga a la relación estructura-proceso, remitiendo el carácter de funcional a este último término. De manera contradictoria, lo funcional también puede remitir a lo fisiológico, esto es, a cierto carácter físico del cuadro, en oposición, quizá, a las cualidades predominantemente psicológicas o cognitivas de otros cuadros. Por último, el autor ubica una cuarta acepción del término, caracterizándolo como un concepto genérico - comodín-que implicaría una referencia difusa al sistema nervioso.

Así pues, teniendo en cuenta que el carácter de "funcional" sirvió de fundamento para la descripción de las neurosis como entidades nosológicas más o menos delimitadas, y descartando la homogeneidad o simplicidad semántica de tal variable, Køppe (2009) sostiene que debe considerarse meramente como un término clasificatorio, ya que puede rastrearse una gran variación de definiciones entre los psiquiatras y neurólogos del siglo xix, lo que dificulta la delimitación de un síntoma central para tal concepto.

No obstante, es de destacar que el estudio de las neurosis fue expandiéndose cada vez más en el campo de la medicina occidental, ocupando un lugar importante en la disciplina psiquiátrica. El pasaje de la neurología a la psiquiatría fue acompañado por un cambio en su concepción, que pasó de ser una enfermedad grave de los nervios, a considerarla como trastornos psiquiátricos menos severos. Aun así, tal distinción también es relativa.

\section{NOSOLOGÍA DE LAS NEUROSIS Y LA ANSIEDAD}

En el vasto terreno nosológico que integra la primera definición de las neurosis se nos hace complejo relacionar esta categoría con la ansiedad. A partir de finales del siglo xix se configuran como representante arcaica de los actuales trastornos mentales y, en especial, de la vertiente patológica de la ansiedad y la angustia.

De todas maneras, en la categoría ideada por Cullen - en especial las que se refieren a las obras de Thomas Willis y Sydenham- se encontraban bajo el ala de las neurosis cuadros tales como la histeria, la hipocondría y la melancolía, a la vez que referencias a síntomas incluidos en descripciones contemporáneas de la ansiedad como disnea, dispepsia o palpitaciones. Por ende, nos encontramos con un término que, llegado el caso, y a pesar de su heterogeneidad, guarda una relativamente cercana correspondencia con lo que consideramos hoy por hoy trastornos mentales y, en especial, con el espectro ansiedad-depresión.

Así, por ejemplo, el médico francés Paul Briquet (1796-1881) definía a la histeria como una neurosis del encéfalo, cuya sintomatología podía incluir constricción epigástrica, opresión torácica palpitaciones, sofocación, mareos y agitación (Conti \& Stagnaro, 2007). Esto es, manifestaciones que nos refieren a aquellas atribuibles a la ansiedad o a la angustia, como ya se mencionó en el punto anterior. Por su parte, JeanMarie Charcot (1825-1893) hace referencia a la neurastenia histérica masculina; la ejemplifica a partir de un caso clínico de un sujeto que refiere síntomas tales como sensación de ahogo, tinitus, palpitaciones, tambaleos, alteraciones visuales y sensación de desmayo (Conti \& Stagnaro, 2007). 
Es necesario destacar, además, la referencia encontrada a tal asociación en la obra de Freud, ya que se evidencia una estrecha relación entre la sintomatología ansiosa y el concepto de neurosis. Sin ir más lejos, desde sus primeras obras este autor se refiere a distintos cuadros - como la histeria, la neurosis obsesiva, la histeria de angustia, la neurosis de angustia y la neurastenia-, los cuales agrupa en las categorías de neurosis actuales y psiconeurosis. Según Vallejo Ruiloba y Gastó Ferrer (2000), la obra freudiana resulta novedosa y revolucionaria por agregar una variable simbólica a la concepción de la neurosis, así como también por darle un sustento teórico unificador para su explicación etiopatogénica.

La "neurosis de ansiedad" o "neurosis de angustia" - cuya descripción más célebre fue realizada por Freud a finales del siglo xix - fue, hasta la década de 1980, el diagnóstico por excelencia en torno a la ansiedad. Sin embargo, la generalidad y poca especificidad del término "neurosis", sumado a los desarrollos en torno a las bases biológicas, psicológicas y sociales de la ansiedad - que privilegiaban una clasificación más descriptiva que conceptual para las investigaciones-, hicieron que sea finalmente removido de la tercera edición del manual diagnóstico de la Asociación Psiquiátrica Americana (APA, 1980), así como de sus posteriores ediciones. Sin embargo, los trastornos neuróticos siguen siendo una categoría actual y válida e íntimamente relacionada con la ansiedad y las fobias, según la décima edición de la Clasificación Internacional de las Enfermedades de la Organización Mundial de la Salud (oms, 1992). Asimismo, en el caso particular de Argentina - como así de otros países de América Latina-, la amplia aceptación y difusión del psicoanálisis hizo de "las neurosis" uno de los conceptos más utilizados tanto en el campo específico de las disciplinas "psi", como en el contexto cotidiano.

La heterogeneidad sintomática de las descripciones de estos cuadros, sumada tanto a las limitaciones como a los avances en relación con los métodos e instrumentos diagnósticos, permitió que sea posible rastrear los antecedentes de la ansiedad en el seno de una gran diversidad de disciplinas biomédicas. En efecto, tal como lo plantea Berrios, antes de formar parte de un concepto más o menos unitario, los síntomas de ansiedad aparecían en áreas tan disímiles como la cardiovascular, gastrointestinal, del oído interno, o neurológica; esto puede evidenciarse en el hecho de que hasta finales del siglo xix los trabajos en relación con ella se encontraban en revistas médicas, y no específicamente psiquiátricas (Berrios, 2008). Algunos de los cuadros que pueden funcionar como antecedentes de los trastornos de ansiedad tal como los conocemos en la actualidad son delirio emotivo (délire emotif), descrito por Bénedict-Augustin Morel (1809-1873) a fines del siglo XIX; neuropatía cerebro-cardiaca, propuesta por Krishaber en 1873; agorafobia, introducido y descrito por Carl Otto Westphal (1833-1890) en 1871; neurastenia, término utilizado por George Beard para designar un estado crónico de agotamiento físico y mental, acompañado por síntomas vagos y generalizados de disconfort y disfunciones corporales; corazón irritable (o síndrome de Da Costa) y astenia neurocirculatoria, cuadros asociados con la medicina militar durante la guerra civil estadounidense.

\section{CONCLUSIONES}

Hemos podido ver que la noción de ansiedad conlleva una complejidad que parte desde su misma delimitación como vocablo y, en consecuencia, como concepto. Al mismo tiempo, es evidente que la de neurosis y las manifestaciones asociadas han podido abarcar una gran diversidad de campos dentro de la biomedicina, y han generado distintas especulaciones en torno a su posible explicación, los aspectos implicados en sus causas, así como las estrategias terapéuticas que podrían ser útiles a la hora de pensar un tratamiento. A su vez, hemos podido observar que a pesar de que las neurosis - y su heredera por excelencia, la ansiedad-, desde mediados del siglo $\mathrm{xx}$, han sido objeto de estudio casi exclusivo de la psiquiatría y la psicología, su modo de expresión fundamentalmente somática las hacían parte de ramas biomédicas tales como la neurología o la cardiología. De todas maneras, las neurosis se transformaron en uno de los bastiones más importantes de las distintas tradiciones psicológicopsiquiátricas, conservando asimismo la característica fundamental de incluir en sus definiciones una gran cantidad de posibles manifestaciones o síntomas. Tal multiplicidad puede observarse, además, en los distintos cuadros descritos, desde siglos atrás, que se derivan o relacionan con estas primeras acepciones del 
término. Ellos contienen, asimismo, los antecedentes de ciertos cuadros psicopatológicos actuales — según las nosografías vigentes-, en especial en lo que hace al énfasis en ciertas manifestaciones o síntomas, como por ejemplo, aquellos ligados al terreno cardiovascular y respiratorio. La diversidad sintomática de estos cuadros mórbidos se refleja, asimismo, en la multiplicidad de teorías que han intentado explicar los fenómenos ansiosos, las cuales van desde explicaciones biológicas hasta elucubraciones sobre procesos inconscientes.

Esta característica de la ansiedad y sus antecedentes conceptuales, muchas veces ha sido invisibilizada por algunas investigaciones, que presentan al constructo ansiedad como un fenómeno discreto y específico. En efecto, en varias ocasiones, en distintos ámbitos, se califican ciertas conductas como "ansiosas", sin especificar qué se entiende por ansiedad, dejando de lado, justamente, las características polisémicas del constructo.

Destacamos la importancia de estas cuestiones, en particular, en aquellos contextos donde es necesa-

\section{REFERENCIAS}

American Psychiatric Association (APA) (1980). Diagnostic and statistical manual of mental disorders (3a.ed.). Washington, DC: APA.

American Psychiatric Association (APA) (2000). Manual diagnóstico y estadístico de los trastornos mentales. (4a. ed.). Barcelona: Masson.

Balint, G. P., Buchanan, W. W. \& Dequeker, J. (2006). A brief history of medical taxonomy and diagnosis. Clinical Rheumatology, 25: 132-135. doi: 10.1007/ s10067-004-1051-z

Barlow, D. H. (2002). Anxiety and its disorders: The nature and treatment of anxiety and panic (2nd ed.). New York: The Gilford Press.

Beer, D. (1996). The dichotomies: psychosis/neurosis and functional/organic: a historical perspective. History of Psychiatry, 7(26): 231-255. doi:10.1177/0957154X96 00702603

Berrios, G. (2008). Historia de los sintomas de los trastornos mentales: la psicopatología descriptiva del siglo XIX. México: Fondo de Cultura Económica.

Cannon, W. B. (1927). Bodily changes in pain, hunger, fear, and rage: an account of recent researches into the func- ria la interacción de distintos profesionales del campo de la salud - psicólogos, psiquiatras, entre otros- que adscriban y manejen diferentes marcos teóricos. A la vez, resulta de particular interés en aquellos países en los que categorías como angustia y ansiedad puedan ser utilizados indistintamente, como es el caso de los países hispanoparlantes. Puesto que esta indiferenciación es evidente en los sistemas diagnósticos operativos más extensamente utilizados, como son el DSM y la CIE, la utilización de uno u otro término puede generar confusiones, no sólo en los profesionales del campo de la salud, sino también en los pacientes diagnosticados. De esta manera, proponemos pasar de una perspectiva centrada en la simplificación de un concepto -en este caso, la ansiedad-, a otra que lo conciba como una construcción histórica y culturalmente situada, que describe determinadas manifestaciones de la psique y el comportamiento humanos, y que necesita una especial atención a la hora de ser utilizada. •

tion of emotional excitement. New York: D. Appleton and Company.

Conti, N. A. \& Stagnaro, J. C. (2007). Historia de la ansiedad: textos escogidos. Buenos Aires: Polemos.

Cullen, W. (1783). Synopsis nosologiae methodicae: sistens morborum classes genera et species. New York: Abraham Hodge.

Cullen, W. (1808). Methodical system of nosology. Stockbridge: Cornelius Sturtevant.

Ey, H. (2008). Estudios psiquiátricos, Vol. 1. Buenos Aires: Polemos.

Freud, S. (1999). Sobre la justificación de separar de la neurastenia un determinado síndrome en calidad de "neurosis de angustia". En Obras completas, Vol. III (pp. 85-115). Buenos Aires: Amorrortu.

Hardy, M. C., Hardy, P. \& Kerneis, O. (1985). Encyclopédie Méd. Chir. Paris: EMc.

Hsu, E. (1999). The transmission of chinese medicine. Cambridge: Cambridge University Press.

Jablensky, A. (1985). Approaches to the definition and classification of anxiety and related disorders in european psychiatry. En A. H. Tuma \& J. D. Maser 
(Eds.), Anxiety and the anxiety disorders (pp. 735758). Hillsdale: Erlbaum.

Jaspers, K. (1998). Psicopatología general. México: Fondo de Cultura Económica.

Køppe, S. (2009). Neurosis: aspects of its conceptual development in the nineteenth century. History of Psychiatry, 20(1), 27-46. doi:10.1177/0957154X08092426

Kristensen, A. S., Mortensen, E. L. \& Mors, O. (2009). The structure of emotional and cognitive anxiety symptoms. Journal of Anxiety Disorders, 23, 600-608. doi:10.1016/j.janxdis.2009.01.009

Lewis, A. (1980). Problems presented by the ambiguous word "anxiety" as used in psychopathology. En G. D. Burrows \& B. Davies (Eds.), Handbook of studies on anxiety (pp. 105-121). Amsterdam: Elsevier/NorthHolland.

Organización Mundial de la Salud (oms). (1992). Décima revisión de la clasificación internacional de las enfermedades: Trastornos mentales y del comportamiento (CIE 10). Versión multiaxial para adultos. Madrid: Meditor.
Papakostas, Y., Eftychiadis, A., Papakostas, G. \& Christodoulou, G. N. (2003). A historical inquiry into the appropriateness of the term "panic disorder". History of Psychiatry, 14(2), 195-204. doi: 10.1177/0957154X030142004

Pichot, P. (1999). The semantics of anxiety. Hum. Psychopharmacol. Clin. Exp., 14, S22-S28. doi:10.1002/ (SICI) 1099-1077(199908)14:1+<S22::AID HUP114>3.0.CO;2-V

Spielberger, C., Gorsuch, R. \& Lushene, R. (1970). Manual for the State-Trait Anxiety Self-Evaluation Questionnaire. Palo Alto: Consulting Psychologist Press.

Strachey, J. (1999). Nota introductoria a "Sobre la justificación de separar de la neurastenia un determinado síndrome en calidad de 'neurosis de angustia". En S. Freud, Obras completas: Primeras publicaciones psicoanalíticas, Vol. III (pp. 83-84). Buenos Aires: Amorrortu.

Vallejo Ruiloba, J. \& Gastó Ferrer, C. (2000). Trastornos afectivos: ansiedad y depresión (2da. ed.). Barcelona: Masson. 